\title{
Herbal Extract from Codonopsis pilosula (Franch.) Nannf. Enhances Cardiogenic Differentiation and Improves the Function of Infarcted Rat Hearts
}

\author{
Jieh-Neng Wang ${ }^{1, *(\mathbb{D})}$, Chung-Dann Kan ${ }^{2, *}$, Lain-Tze Lee ${ }^{3}$, Lynn L. H. Huang ${ }^{4} \mathbb{D}$, Ya-Li Hsiao ${ }^{2}$, Allen H. Chang ${ }^{4}$, \\ Wanchun Liu ${ }^{3}$, Cheng $\operatorname{Lin}^{3}$ and Chou-Wen Lin ${ }^{3,5, *}$
}

1 Department of Pediatrics, National Cheng Kung University Hospital, College of Medicine, National Cheng Kung University, Tainan 70401, Taiwan

2 Department of Surgery, National Cheng Kung University Hospital, College of Medicine, National Cheng Kung University, Tainan 70401, Taiwan; bsmlily@gmail.com

3 Herbal Medical Product Technology Division, Biomedical Technology and Device Research Laboratories, Industrial Technology Research Institute, Hsinchu 31057, Taiwan; lens@gp-corp.com (L.-T.L.); wanchunliu@itri.org.tw (W.L.); chenglin@gp-corp.com (C.L.)

4 Department of Biotechnology and Bioindustry Sciences, National Cheng Kung University, Tainan 70401, Taiwan; lynn@mail.ncku.edu.tw (L.L.H.H.); sparktrix@gmail.com (A.H.C.)

5 Gold Medal Glycine Tomentella Hayata Biological Technology Company, Tainan 73052, Taiwan

* Correspondence: jiehneng@mail.ncku.edu.tw (J.-N.W.); kcd5086@gmail.com (C.-D.K.); cwlin2002@yahoo.com (C.-W.L.)

check for updates

Citation: Wang, J.-N.; Kan, C.-D.; Lee, L.-T.; Huang, L.L.H.; Hsiao, Y.-L.; Chang, A.H.; Liu, W.; Lin, C.; Lin, C.-W. Herbal Extract from Codonopsis pilosula (Franch.) Nannf. Enhances Cardiogenic Differentiation and Improves the Function of Infarcted Rat Hearts. Life 2021, 11, 422. https://doi.org/10.3390/life11050422

Academic Editor: Yi Dai

Received: 21 March 2021

Accepted: 2 May 2021

Published: 5 May 2021

Publisher's Note: MDPI stays neutral with regard to jurisdictional claims in published maps and institutional affiliations.

Copyright: (c) 2021 by the authors. Licensee MDPI, Basel, Switzerland. This article is an open access article distributed under the terms and conditions of the Creative Commons Attribution (CC BY) license (https:/ / creativecommons.org/licenses/by/ $4.0 /)$.

\begin{abstract}
Background: The roots of Codonopsis pilosula (Franch.) Nannf. have been used in traditional Chinese medicine for treating cardiovascular disease. In the current study, we aimed to discover herbal extracts from C. pilosula that are capable of improving cardiac function of infarcted hearts to develop a potential therapeutic approach. Methods: A mouse embryonic stem (ES) cell-based model with an enhanced green fluorescent protein (eGFP) reporter driven by a cardiomyocyte-specific promoter, the $\alpha$-myosin heavy chain, was constructed to evaluate the cardiogenic activity of herbal extracts. Then, herbal extracts from C. pilosula with cardiogenic activity based on an increase in eGFP expression during ES cell differentiation were further tested in a rat myocardial infarction model with left anterior descending artery (LAD) ligation. Cardiac function assessments were performed using echocardiography, 1, 3, and 6 weeks post LAD ligation. Results: The herbal extract 417W from C. pilosula was capable of enhancing cardiogenic differentiation in mouse ES cells in vitro. Echocardiography results in the LAD-ligated rat model revealed significant improvements in the infarcted hearts at least 6 weeks after $417 \mathrm{~W}$ treatment that were determined based on left ventricle fractional shortening (FS), fractional area contraction (FAC), and ejection fraction (EF). Conclusions: The herbal extract 417W can enhance the cardiogenic differentiation of ES cells and improve the cardiac function of infarcted hearts.
\end{abstract}

Keywords: herbal extracts; cardiogenesis; cardiomyocytes; myocardial infarction

\section{Introduction}

Heart failure (HF) is a frequent complication of myocardial infarction (MI) [1]. Many acute and chronic adaptations occur due to MI that progresses to HF, for example, neurohumoral hyperactivity, oxidative stress, inflammatory response, and cardiac remodeling [2]. According to pathophysiology, myocardial ischemia leads to ultrastructural damage, contractility dysfunction, an increase in reactive oxygen species (ROS) production, and changes in myocardial metabolism which culminate in necrosis of cells [3]. Mitochondrial dysfunction is a hallmark of heart failure, and a marker of both oxidative stress and inflammation. Myocardial injury leads to activation of a stereotyped inflammatory cascade comprised of early neutrophil ingress followed by monocyte-macrophage infiltration [4]. Recent studies 
have revealed oxidative stress and inflammation to be key pathophysiological elements of heart failure syndrome. Oxidative stress and inflammation are closely connected to each other, both in the acute phase after myocardial infarction and during chronic cardiac remodeling [5]. The precise contribution of the different pathophysiological components (e.g., microvascular dysfunction and inflammation) to injury is likely to be heterogeneous, and therefore understanding mechanistic pathways in specific patient subgroups is key to identifying novel therapeutic strategies [6].

However, current standard treatments for $\mathrm{HF}$, including pharmacological and surgical interventions, have their limitations. These therapeutic interventions cannot restore damaged heart tissues and are always associated with side effects [7]. As such, alternative treatments that can restore injured myocardium have been the current focus of HF intervention. Among the emerging alternative strategies for treating HF, stem cells (including ES cells), induced pluripotent stem (iPS) cells, and multipotent adult stem cells have shown promise [8-10]. These stem cells can be coaxed in vitro into cardiac progenitors which, then, differentiate into cardiomyocytes that replace infarcted cardiac tissues and eventually restore normal heart function. However, the potential risk of neoplastic transformation from the transplantation of unguided non-cardiac progenitors has remained a concern [11]. Other challenges to this type of stem cell therapy include expansion of appropriate stem cell populations, restricted homing, loss of the majority ( $95 \%$ ) of infused stem cells, functional integration of transplanted stem cells, and delivery strategies [7].

Another therapeutic strategy for HF treatment has been to initiate a cardiogenic activity in vivo by promoting myocardial differentiation of circulated stem cells or by activating the proliferation and differentiation of resident cardiac stem or progenitor cells to regenerate new heart tissues. This strategy requires no invasive therapeutic interventions for infarcted myocardium treatment. Although the fundamental mechanism underlying cardiogenesis has not been fully elucidated, some laboratories have searched for molecules with cardiogenic activity that may serve therapeutic purposes in patients with MI [12-15].

While there is a strong conceptual framework for antioxidant or anti-inflammatory strategies as adjunctive therapies for the treatment of heart failure, these drugs have only been evaluated in a few clinical trials [5]. Recent studies have shown that Chinese herbal medicines, widely utilized for thousands of years in Asian countries, possess antiinflammatory and antioxidant functions that can help with cardiovascular diseases [16-18]. Codonopsis pilosula is a perennial flowering plant species native to Northeast Asia and Korea that usually grows around stream banks and forest openings under the shade of trees. The roots of C. pilosula (radix) have been used in traditional Chinese medicine to lower blood pressure, increase red and white blood cell counts, cure appetite loss, strengthen the immune system, and replenish chi [19]. Moreover, studies have shown some potential benefits for patients with AMI, such as reduced cardiac death and HF, although the precise mechanisms have remained unclear [20-22]. Therefore, we hypothesized that the herbal extract derived from the water-solubilized fraction of Codonopsis pilosula (Franch.) Nannf. could contain cardiogenic activity and improve cardiac function of infarcted rat hearts.

\section{Materials and Methods}

\subsection{Vector}

The mouse $\alpha$-myosin heavy chain ( $\alpha$-MHC) promoter was generously provided by Jeffrey Robbins [23], while the DNA fragment of the BamHI-SalI $\alpha$-MHC promoter (5.5-kb fragment) was inserted into the BglII-SalI site of the multiple cloning site of pEGFP-1 (Clontech, Palo Alto, CA, USA) to form pMGN22 for the transfection of ES cells.

\subsection{Embryonic Stem Cell Lines}

A transgenic ES cell line was created by transfecting pMGN22 into mouse ES-D3 cells (ATCC) using Lipofectamine 2000 (Invitrogen, Carlsbad, CA, USA), as described by the manufacturer. Stable transgenic ES cells, EMG8, were selected in the presence of $500 \mu \mathrm{g} / \mathrm{mL}$ G418 (Sigma, St. Louis, MO, USA) on a feeder cell-free culture plate coated 
with $0.1 \%$ gelatin (Millipore, Billerica, MA, USA) in ES medium containing Dulbecco's Modified Eagle's Medium (Gibco, Paisley, Scotland, UK), $0.1 \mathrm{mM}$ non-essential amino acids (Gibco), $0.15 \mathrm{mM} \alpha$-monothioglycerol (ICN Biomedicals Inc., Costa Mesa, CA, USA), 15\% ES cell-qualified fetal bovine serum (Gibco), penicillin G (100 U/mL), $100 \mu \mathrm{g} / \mathrm{mL}$ streptomycin, $250 \mathrm{ng} / \mathrm{mL}$ amphotericin B (Sigma), and $10^{3} \mathrm{U} / \mathrm{mL}$ ESGRO (Chemicon, Temecula, CA, USA). Then, EMG8 cells were cultured in ES medium containing $250 \mu \mathrm{g} / \mathrm{mL}$ $\mathrm{G} 418$ in a $5 \% \mathrm{CO}_{2}$ environment at $37^{\circ} \mathrm{C}$, and the medium was changed every other day.

\subsection{Embryonic Stem Cell Differentiation}

To initiate spontaneous differentiation of mouse ES cells, EMG8 cells were seeded at a density of 2000 cells/well in a 96-well plate (Corning, Corning, New York, NY, USA) coated with $0.1 \%$ gelatin (Millipore) in ES differentiation medium containing high-glucose Dulbecco's Modified Eagles's Medium (Gibco), $0.1 \mathrm{mM}$ non-essential amino acids (Gibco), $0.1 \mathrm{mM} \beta$-mercaptoethanol (Sigma), 20\% fetal bovine serum (Gibco), $100 \mathrm{U} / \mathrm{mL}$ penicillin $\mathrm{G}$ (Gibco), $100 \mu \mathrm{g} / \mathrm{mL}$ streptomycin (Gibco), $250 \mathrm{ng} / \mathrm{mL}$ amphotericin B (Sigma), and $250 \mu \mathrm{g} / \mathrm{mL}$ G418 (Sigma). The medium was replaced every other day, and the enhanced green fluorescent protein (eGFP) intensity was measured after 10 days using a SpectraMax M2 microplate reader (Molecular Devices, Sunnyvale, CA, USA) at excitation and emission wavelengths of 488 and $519 \mathrm{~nm}$, respectively. However, spontaneous differentiation of EMG8 cells were also conducted through the formation of embryoid bodies (EBs) [24,25]. Briefly, the EB was formed in a hanging drop containing 500 cells in $25 \mu \mathrm{L}$ of ES differentiation medium on the cover of a petri dish (SPL) and cultured in a cell culture incubator with $5 \%$ $\mathrm{CO}_{2}$ at $37^{\circ} \mathrm{C}$ for two days, followed by resuspension onto petri dishes in ES differentiation medium for another 5 days of growth. Then, EBs were transferred to gelatin-coated 6-well plates (Nunc, Roskilde, Denmark ) in ES differentiation medium for continuous differentiation to examine contractile cell foci and eGFP fluorescence. Images of the contractile EB outgrowths expressing eGFP were taken using a Leica DM IRBE microscope (Leica Microsystems, Heidelberg, Germany).

\subsection{Preparation of Herbal Extracts}

The herb used in this study was acquired from a farm in Hubei province, China. A total of $100 \mathrm{~g}$ of the herb powder was extracted in $1 \mathrm{~L}$ of water at $95^{\circ} \mathrm{C}$ using the hot backflow extraction method for $2.5 \mathrm{~h}$. After cooling the crude extract, the clear extract was separated from the crude extract either by a Whatman No.1 filter (Whatman International, Maidstone, UK) with suction or by centrifugation, and then concentrated, frozen, and lyophilized to create a dry powder for long-term storage. To evaluate cardiogenic activity, the herbal extract was re-dissolved in $\mathrm{ddH}_{2} \mathrm{O}$ to a concentration of $100 \mu \mathrm{g} / \mathrm{mL}$.

\subsection{Immunocytochemical Staining}

EMG8-derived EB outgrowths seeded on gelatin-coated glass slides after 15 days of differentiation were fixed with $4 \%$ formaldehyde at room temperature for $20 \mathrm{~min}$, permeabilized with $0.5 \%$ Triton X-100 (Sigma) for $5 \mathrm{~min}$, and then blocked with $5 \%$ normal goat serum (NGS) in PBS at room temperature for $20 \mathrm{~min}$. Then, EB outgrowths were incubated with primary antibody (rabbit anti-Nkx2.5,1:150 dilution, GeneTex, Irvine, CA, USA) or mouse anti- $\alpha$-actinin IgG1 (1:200 dilution, Enzo, Farmingdale, NY, USA) in $1 \%$ NGS/PBS at $4{ }^{\circ} \mathrm{C}$ overnight. After washing twice with PBS, EB outgrowths were exposed to secondary antibodies ((goat-anti-rabbit Qdot655-conjugated IgG, 1:200 dilution, Invitrogen, Carlsbad, CA, USA) or goat-anti-mouse TRICT-conjugated IgG (1:200 dilution, Jackson ImmunoResearch, West Grove, PA, USA)) at room temperature for $1 \mathrm{~h}$. Then, images of immunostained cells were taken using a Leica DM IRBE microscope (Leica Microsystems, Heidelberg, Germany). 


\subsection{Effects of Herbal Extracts on Differentiating EMG8 Cells}

To test which herbs had the potential cardiogenic activity, more than 100 herbal extracts were prepared from our database (data not shown). Here, only six herbal extracts (0041W, Bupleurum chinense; 148W, Lobelia chinensis; 154W, Lophatherum gracile; 165W, Lithospermum erythrorhizon; 239W, Forsythia suspense; and 417W, Codonopsis pilosula (Franch.)) are shown as the representatives from many other tests. In addition, ascorbic acid (Vitamin C), which was reported to enhance differentiation of ES cells into cardiomyocytes [14], was used as a positive control. To determine the cardiogenic activity of herbal extracts, undifferentiated EMG8 cells were seeded onto a gelatin-coated 96-well plate at 2000 cells/well in ES differentiation medium. Herbal extracts were added to each well on Day 2, with five replicates for each sample, at a concentration of $0.5 \mathrm{mg} / \mathrm{ml}$. On Day 11, the medium on each well was removed, and the fluorescence was analyzed in a microplate reader at excitation and emission wavelengths of 488 and $519 \mathrm{~nm}$, respectively. Undifferentiated EMG8 cells were subjected to spontaneous differentiation in the presence of different herb extract dosing were also tested. The eGFP fluorescence intensities were measured on Day 9 and were presented as the mean value of five replicates.

\subsection{Experimental Animals}

Male inbred Wistar rats (3 months old, weighing 250-300 grams, Animal Center of the National Cheng Kung University Medical College) were used as the experimental animals. The were six rats in the control group, and the same number of rats was in the test group. All animal procedures were carried out with the approval of the Animal Care Committee of the National Cheng Kung University Hospital Research Institute (IACUC approval no. 102179) and in compliance with the Guide for the Care and Use of Laboratory Animals by the Institute of Laboratory Animal Resource. Briefly, all rats were housed in cages at room temperature $\left(20-26{ }^{\circ} \mathrm{C}\right)$ in an atmosphere with $60-70 \%$ humidity and under a $12 / 12 \mathrm{~h}$ light/dark cycle with unrestricted access to water and food.

\subsection{The Rat Myocardial Infarction Model}

After the induction of anesthesia through isoflurane inhalation in an anesthetic chamber, rats were intubated with a $16-\mathrm{G}$ angiocatheter and ventilated with positive-pressure ventilation using a Harvard ventilator. Anesthesia was maintained with $2 \%$ inhaled isoflurane (Panion \& Bf Biotech, Taoyuan, Taiwan), and electrocardiograph (EKG) monitoring was established. A left anterior thoracotomy was performed, after which the heart was visualized from the fourth intercostal space through a pericardial incision. Then, the left anterior descending artery (LAD) was encircled and ligated with a 7-0 proline stitch, as previously reported [26]. Myocardial ischemia was confirmed through visual inspection and EKG ST-segment elevation during the operation. The ribs and subcutaneous and skin incisions were closed with 3-0 Vicryl (Ethicon Co, Inc., Sommerville, NJ, USA) and 3-0 Ethilon (Ethicon $\mathrm{Co}$, Inc) in layered sutures. Rats received identical amounts of intramuscular analgesia (meperidine, $0.3-0.5 \mathrm{mg} / 100 \mathrm{~g}$ body weight) for pain control after surgery.

\subsection{Effects of Herbal Extracts in the Rat Myocardial Infarction Model and Cardiac Function Assessment}

Further tests for the cardiogenic effect of herbal extracts were conducted in an in vivo animal model. One day after LAD ligation, the rats were divided into the following two groups: the control group $(n=6)$ which received normal saline, and the test group $(n=6)$ which received $10 \mathrm{mg}$ of herbal extracts/rat dissolved in $100 \mu \mathrm{L}$ of distilled water daily for 10 consecutive days by intraperitoneal injection (IP). After 1, 3, and 6 weeks, cardiac function and affected wall size were measured using echocardiography to collect data. Rats were anesthetized and placed in the left-lateral decubitus position. Short-axis two-dimensional images at the mid-papillary level of the left ventricle (LV) were stored as digital loops, and both the end-systolic (ESA) and end-diastolic (EDA) cavity areas were determined by tracing the endocardial borders. The fractiona shortening (FS) was 
calculated from M-mode images as ((LVEDD - LVESD)/LVEDD) × 100, where LVEDD represents the LV end-diastolic dimension and LVESD represents the LV end-systolic dimension. The FAC was calculated as $((E D A-E S A) / E D A) \times 100$, while the EF was calculated as $(\mathrm{SV} / \mathrm{EDV}) \times 100$, where $\mathrm{SV}$ is the stroke volume and is equal to end-diastolic volume (EDV) minus the end-systolic volume.

Measurements were taken three times, the average of which was used for analysis. After positive LAD ligation determined through echocardiography according to the random digits tables, rats were randomized into two study groups, i.e., the control group and the herbal extracts treatment group. Thereafter, an observer blinded to the study groups repeated the cardiac function assessment using echocardiography. The animal study protocol is shown below (Figure 1).
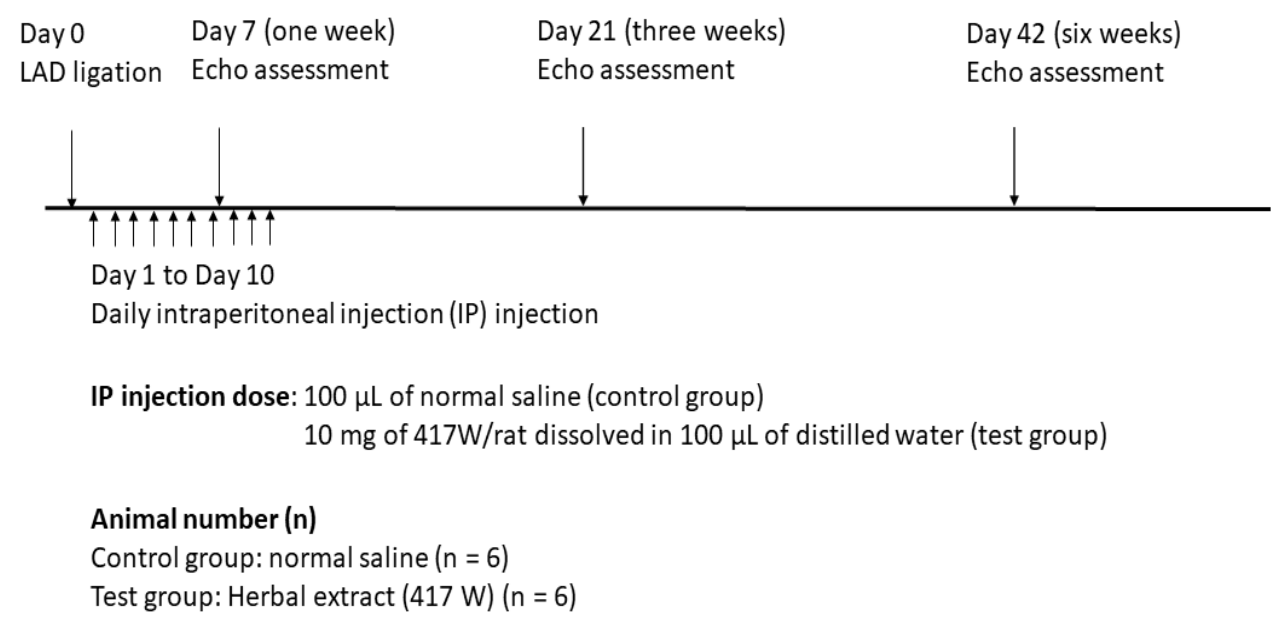

Figure 1. Animal study protocol.

\section{Results}

3.1. Cardiomyocyte Identity of Enhanced Green Fluorescent Protein (eGFP)-Expressing EB Outgrowths

To establish the ES cell-based model for evaluating herbal extracts with cardiogenic activity, transgenic ES cells (EMG8) were created to express eGFP fluorescence under a cardiac-specific $\alpha$-MHC promoter. Given that $\alpha$-MHC is a cardiomyocyte-specific gene expressed early in the developing heart [27], eGFP expression would be associated with cardiomyocytes in differentiating ES cells.

EMG8 cells were subjected to EB formation through the hanging drop method. eGFP expression within EB outgrowths on Day 15 was examined under an epifluorescence microscope. The bright-field image on the left panel of Figure 2A shows the contractile cell foci within the circular area. The green fluorescence of eGFP expression was also detected on the EB outgrowths (middle) and exclusively overlaid with those contractile cardiogenic foci (right panel), suggesting that these contractile cells expressed eGFP.

To further confirm the identity of eGFP-expressing contractile cardiogenic foci, EB outgrowths were subjected to immunocytochemistry. The results presented in Figure $2 \mathrm{~B}$ show that these eGFP-expressing contractile cells were stained with antibodies against both cardiomyocyte-specific proteins, i.e., $\alpha$-actinin and $\mathrm{Nkx2.5}$, further proving that these contractile cells were cardiomyocytes.

\section{2. eGFP Expression on Spontaneous Differentiating EMG8 Cells}

To use the spontaneous differentiation of EMG8 cells directly, without EB formation, as the cell-based model for evaluating cardiogenic activity of herbal extracts, in this study, we also examined whether EMG8 cells could undergo cardiogenic differentiation without the EB formation stage. To this end, EMG8 cells were seeded directly onto a culture plate in the presence of ES differentiation medium. After 10 days, differentiating EMG8 cells were subjected to epifluorescence microscopy to examine eGFP fluorescence expression. Figure 3 
shows the presence of eGFP-positive cells, suggesting that cardiomyocyte formation could also occur through spontaneous differentiation of EMG8 cells without EB formation. Hence, an evaluation of herb extracts with cardiogenic activity could be performed by directly seeding EMG8 cells onto 96-well plates in the presence of herbal extracts.

A
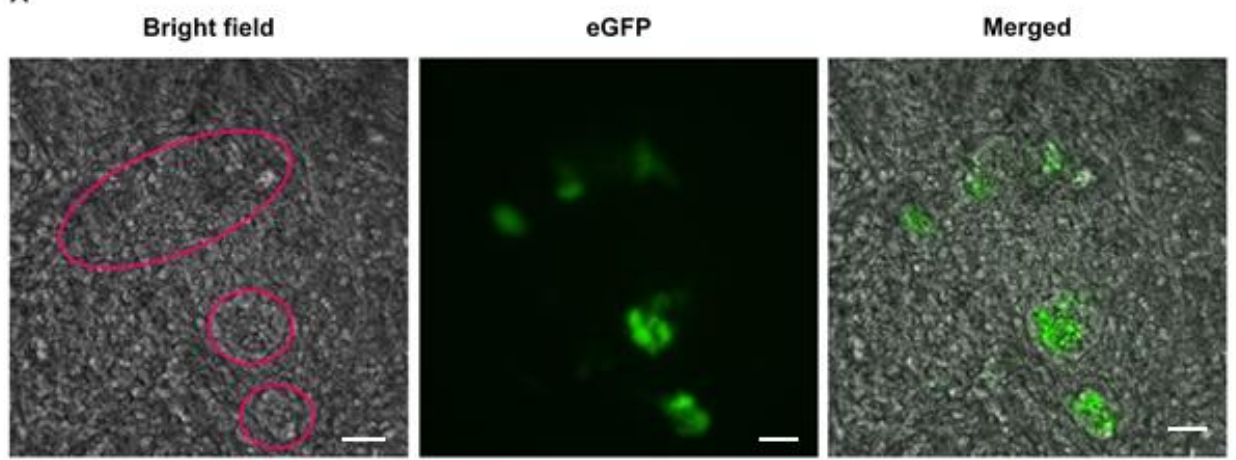

B

\begin{abstract}
Bright field
\end{abstract}

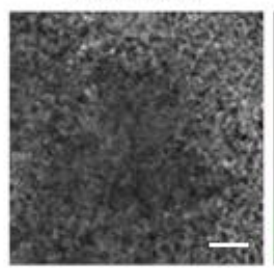

eGFP

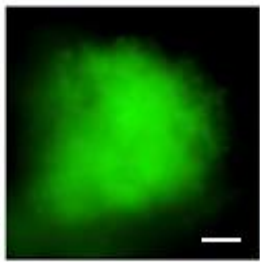

a-Actinin

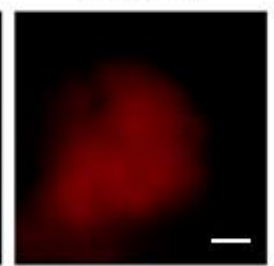

Nkx2.5

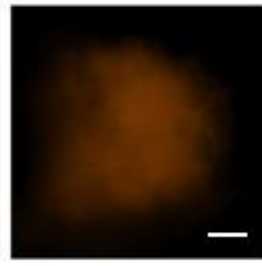

Merged

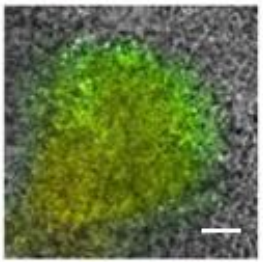

Figure 2. Cardiomyocyte identity of enhanced green fluorescent protein (eGFP)-expressing contractile cardiogenic foci. (A) Contractile cardiogenic foci within the red circles overlaid with eGFP-expressing cells; (B) these EMG8-derived eGFP-expressing contractile cardiogenic foci within EB outgrowths were stained with antibodies against both cardiomyocyte-specific $\alpha$-actinin and Nkx2.5. Bar $=50 \mu \mathrm{m}$ in $\mathrm{A}$, bar $=200 \mu \mathrm{m}$ in $\mathrm{B}$.

Bright field

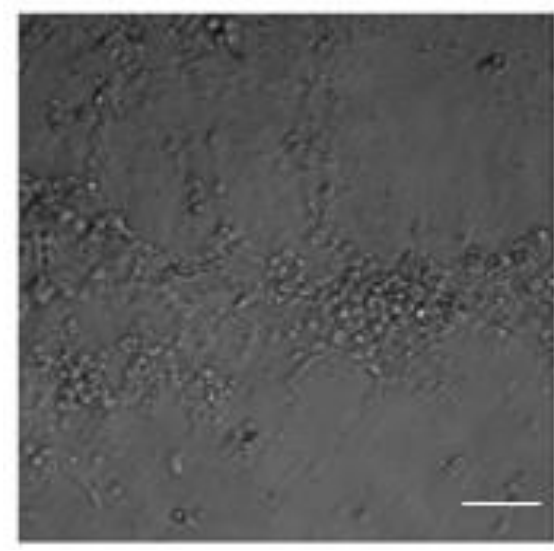

eGFP

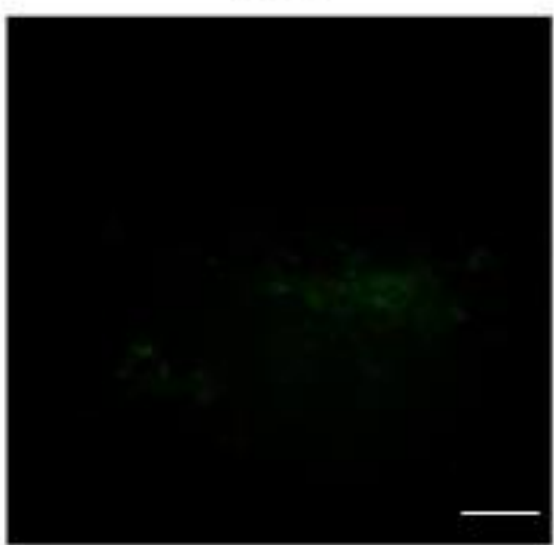

Merged

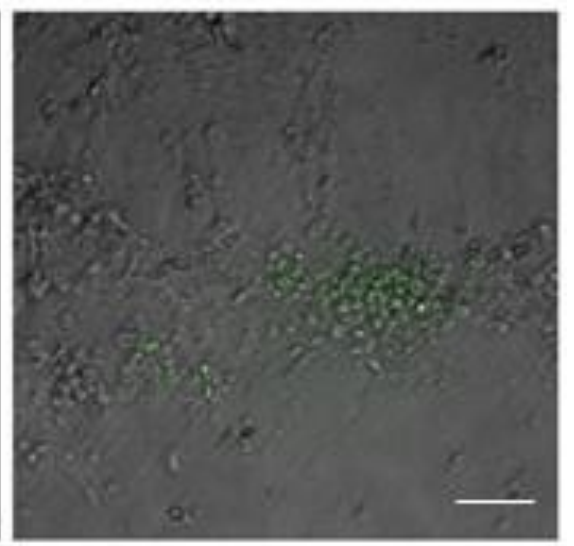

Figure 3. eGFP-expressing cardiomyocytes can be observed among spontaneously differentiating EMG8 cells without EB formation. Bar $=100 \mu \mathrm{m}$.

\subsection{Resul ts of Herbal Extracts on Differentiating EMG8 Cells}

Among the herbal extracts tested, extract 417W, which indicated the water-solubilized extract of Codonopsis pilosula (Franch.) Nannf., showed significant cardiogenic activity that was $24.2 \%$ better than the herbal extracts and the control set, as shown in Figure $4 \mathrm{~A}$. The 417W extract also showed better cardiogenic activity than ascorbic acid (vitamin C). 
Moreover, the cardiogenic differentiation of 417W into EMG8 cells was dose dependent, as shown in Figure 4B.

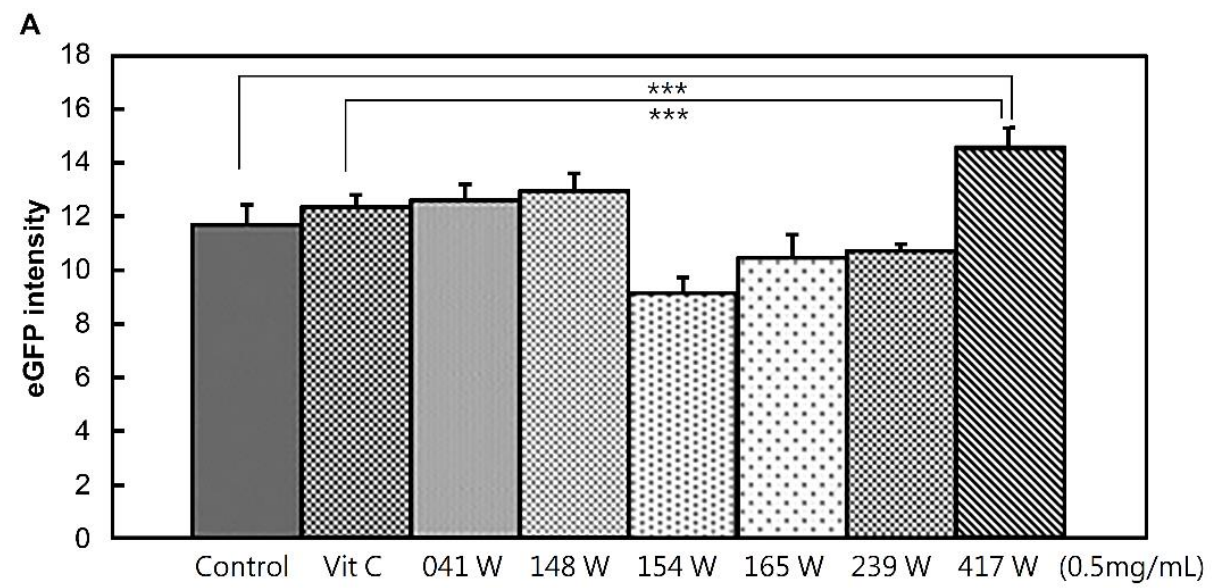

B

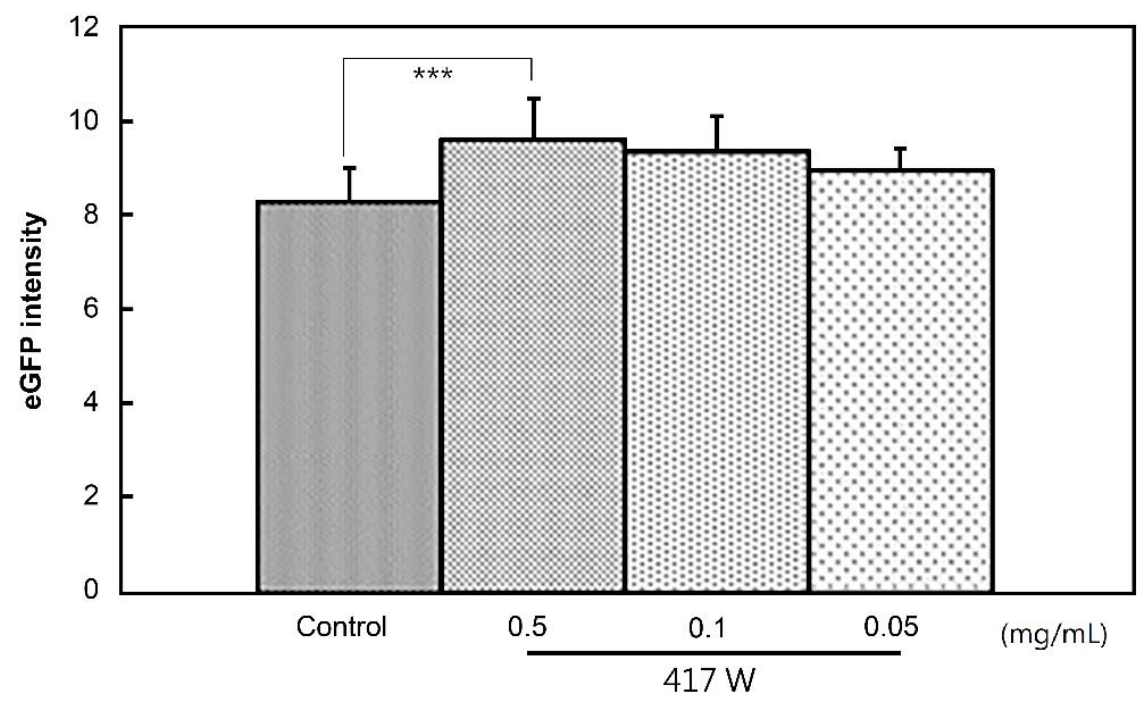

Figure 4. Herbal extract $417 \mathrm{~W}$ exhibited potential cardiogenic activity. (A) Different herbal extracts were added to undifferentiated EMG 8 cells at $0.5 \mathrm{mg} / \mathrm{mL}$. Significant differences were shown using a one-way ANOVA test. Post hoc two-by-two comparison using Fisher's least significant difference (LSD) demonstrated a difference between $417 \mathrm{~W}$ and vitamin $\mathrm{C}(p<0.001)$ and control $(p<0.001)$ as compared with the other herbal extracts; (B) undifferentiated EMG8 cells were subjected to spontaneous differentiation in the presence of $417 \mathrm{~W}$ at $0.5,0.1$, and $0.05 \mathrm{mg} / \mathrm{mL}$. Student t-test showed that e-GFP intensity was significantly higher in the $417 \mathrm{~W}(0.5 \mathrm{mg} / \mathrm{mL})$ group than in the control group ${ }^{* * *} p<0.001$.

The cardiogenic activity of 417W was also examined using the model of EB formation. Accordingly, 417W promoted more beating EB outgrowths as compared with other herbal extracts, which was consistent with observations using the model of ES cell differentiation without EB formation (data not shown). The aforementioned results suggested that $417 \mathrm{~W}$ could significantly enhance cardiogenic differentiation of undifferentiated EMG8 cells.

\subsection{Results of $417 W$ in the Rat Myocardial Infarction Model}

After 1,3, and 6 weeks, FS, FAC, and EF of the treated hearts were assessed, with the corresponding values being summarized in Table 1 . 
Table 1. Fractional shortening (FS), frctional area contraction (FAC), and ejection fraction (EF) values collected from the control and 417W-treated mice 1, 3, and 6 weeks post left anterior descending artery ligation. Data are expressed as the mean $(\%) \pm$ standard deviation of six replicates.

\begin{tabular}{ccccccc}
\hline \multicolumn{3}{c}{ Control $(\boldsymbol{n = 6 )}$} \\
\hline & 1 week & 3 weeks & 6 weeks & 1 week & 3 weeks & 6 weeks \\
FS (\%) & $26.9 \pm 6.0$ & $21.3 \pm 7.1$ & $24.3 \pm 6.4$ & $25.8 \pm 7.1$ & $30.0 \pm 5.2$ & $29.8 \pm 6.9$ \\
FAC (\%) & $46.9 \pm 8.7$ & $35.7 \pm 8.5$ & $37.4 \pm 11.3$ & $45.2 \pm 9.3$ & $56.8 \pm 9.0$ & $50.6 \pm 8.0$ \\
EF (\%) & $59.4 \pm 19.9$ & $52.2 \pm 12.4$ & $52.1 \pm 13.6$ & $56.8 \pm 9.8$ & $73.2 \pm 9.2$ & $67.5 \pm 7.9$ \\
\hline
\end{tabular}

FS, fractional shortening; FAC, fractional area contraction; $\mathrm{EF}$, ejection fraction.

The echocardiography results, shown in Figure 5, revealed no significant improvement 1 week after $417 \mathrm{~W}$ treatment. However, 3 weeks after treatment, $417 \mathrm{~W}$-treated rats $(n=6)$ showed a 40.8\% improvement in FS ( $p<0.05), 59.1 \%$ improvement in FAC $(p<0.001)$, and $40.2 \%$ improvement in EF $(p<0.005)$ as compared with the control group of LAD-ligated rats $(n=6)$. After 6 weeks, a $22.6 \%$ improvement in FS, 35.3\% $(p<0.05)$ improvement in FAC, and $29.6 \%(p<0.05)$ improvement in EF were noted. Although both FAC and EF in the $417 \mathrm{~W}$-treated group declined from week three to week six post $417 \mathrm{~W}$ treatment, the functional improvement in the infarcted hearts was still considerably more significant than in the control group, thereby confirming that herbal extract $417 \mathrm{~W}$ could enhance the function of infarcted hearts.

A

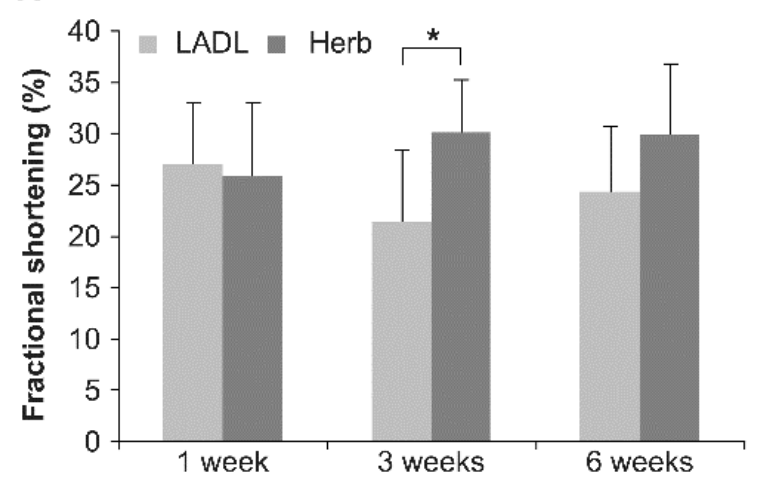

B

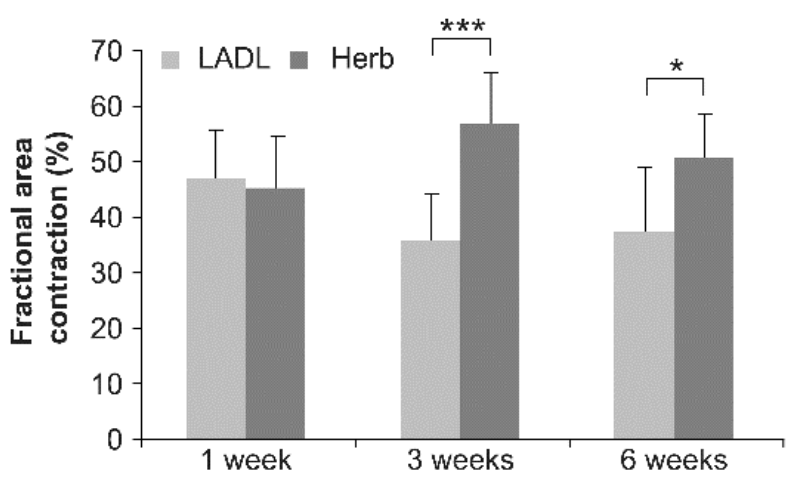

C

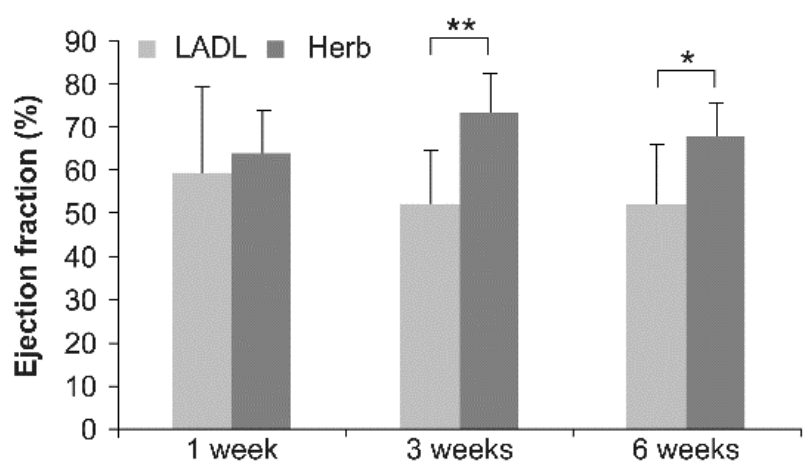

Figure 5. 417W treatment improved cardiac function in left anterior descending artery (LAD)-ligated rats. The FS, FAC, and EF were monitored 1, 3, and 6 weeks post LAD ligation (A, B, and C, respectively). ${ }^{*} p<0.05$, ${ }^{* *} p<0.005$, and ${ }^{* * *} p<0.001$ as compared with the control group.

\section{Discussion}

The current study tested the cardiogenic activity of water-solubilized herbal extracts using a transgenic ES cell-based model. Our in vitro tests found that the water-solubilized extract, 417W from Codonopsis pilosula (Franch.) Nannf., showed cardiogenic activity, sug- 
gesting it has potential for promoting cardiac differentiation of ES cells in vitro. Consistent with this result, the infarcted hearts of the LAD-ligated rat model treated with $417 \mathrm{~W}$ also showed functional improvements in FS, FAC, and EF, although whether these improvements were due to the replenishment of cardiomyocytes derived from stem cells in the circulation, resident cardiac stem cells, or other mechanisms requires further investigation.

The molecular pathways underlying heart formation are complicated and require further exploration. Cardiac differentiation in the embryo originates from the anterolateral mesoderm that receives instructive guidance from the adjacent endoderm [28,29]. Both bone morphogenetic protein and fibroblast growth factor families of signaling molecules expressed in the endoderm of the cardiogenic region are involved in the determination of cardiac fate [30,31], while Nkx2.5, MEF-C, and the GATA family are responsible for initiating cardiomyocyte differentiation [32-34]. Moreover, evidence has shown that noncanonical Wnt/JNK signaling molecules can promote cardiogenesis during embryogenesis and also in adult stem cells [35,36]. Hence, granted that $417 \mathrm{~W}$ acts on the cardiogenic program, these factors could possibly account for its cardiogenic activity.

Over the years, laboratory data have shown that many medicinal herbs, the most famous being Ginseng, Ginkgo biloba, Ganoderma lucidum, and Gynostemma pentaphyllum, may have therapeutic value in cardiovascular diseases (CVD) as they can interfere with several CVD risk factors [37]. Ginseng and its ginsenoside constituents have vasorelaxation, antioxidation, anti-inflammation, and anticancer activities [38]. Codonopsis pilosula (Franch.) Nannf., the root of the Codonopsis genus, has been used for the treatment of various diseases. Other studies reported that Codonopsis pilosula (Franch.) Nannf. regulated gastric basic electrical rhythm disorder under stress conditions and inhibited gastric motility in rats with acute gastric ulcer [39]. It has also been used to revitalize spleen and liver function [40]. Total alkaloids extracted from Codonopsis pilosula (Franch.) Nannf. with $20 \%$ alcohol were able to enhance neurite outgrowth induced by nerve growth factor in PC12 cells [41], indicating that different components of Codonopsis pilosula (Franch.) Nannf. may be responsible for different therapeutic effects. Because the herbal extract protocols are not the same, this $417 \mathrm{~W}$ extract contains many ingredients and has not been studied completely yet. As more studies are being continued, new components from the 417W extract have been discovered, such as saponin, alkaloids, atrctylenolide III, angelicin, and psoralen. Therefore, currently, it is hard to know exactly what possible components $417 \mathrm{~W}$ extract contains, and which component in 417W extract plays the major role on enhancing cardiogenic differentiation. Therefore, the mechanism through which Codonopsis pilosula (Franch.) Nannf. exerts its cardiovascular therapeutic effects still remains unclear. Notably, Tsai et al. [22] found that Codonopsis pilosula (Franch.) Nannf. attenuated the cardiac-impaired insulin-like growth factor (IGF) II receptor pathway in myocardial cells, while Chang et al. [20] reported that it suppressed the apoptotic pathway enhanced by AngII plus Leu27-IGFII in myocardial cells. Therefore, the active components of 417W responsible for cardiogenic activity require further purification and examination before any applications.

\section{Conclusions}

Our study demonstrated that the herbal extract 417W from the water-solubilized fraction of Codonopsis pilosula (Franch.) Nannf. exhibited cardiogenic activity in both the ES cell-based model and the in vivo LAD-ligated rat model. Hence, this screening system can be a useful method for evaluating herbal extracts with cardiogenic activity; however, further studies are needed.

Author Contributions: Conceptualization, J.-N.W., C.-D.K. and C.-W.L.; methodology, C.-D.K. and C.-W.L.; validation, L.-T.L. and Y.-L.H.; formal analysis, Y.-L.H. and L.L.H.H.; investigation, A.H.C.; resources, L.L.H.H.; data curation, Y.-L.H., W.L. and C.-W.L.; writing-original draft preparation, J.-N.W.; writing-review and editing, C.-D.K.; visualization, C.L.; supervision, C.-W.L.; project administration, W.L.; funding acquisition, C.-W.L. All authors have read and agreed to the published version of the manuscript. 
Funding: This research was funded by grant nos. 9356E28200, A356E28200, and B356E22100 from the Industrial Technology Research Institute.

Institutional Review Board Statement: This study was carried out with the approval of the Animal Care Committee of the National Cheng Kung University Hospital Research Institute (IACUC approval no. 102179) and in compliance with the Guide for the Care and Use of Laboratory Animals of the Institute of Laboratory Animal Resource.

Informed Consent Statement: Not applicable.

Data Availability Statement: Data available on request due to institute restrictions.

Acknowledgments: We are grateful to Jeffrey Robbins (Department of Pharmacology and Cell Biophysics University of Cincinnati, College of Medicine, OH, USA) for the generous gift of the $\alpha$-MHC promoter construct.

Conflicts of Interest: The authors declare no conflict of interest.

\section{References}

1. Bahit, M.C.; Kochar, A.; Granger, C.B. Post-Myocardial Infarction Heart Failure. JACC. Heart Fail. 2018, 6, 179-186. [CrossRef] [PubMed]

2. Gabriel-Costa, D. The pathophysiology of myocardial infarction-induced heart failure. Pathophysiology 2018, 25, 277-284. [CrossRef] [PubMed]

3. Shahzad, S.; Hasan, A.; Faizy, A.F.; Mateen, S.; Fatima, N.; Moin, S. Elevated DNA damage, oxidative stress, and impaired response defense system inflicted in patients with myocardial infarction. Clin. Appl. Thromb. Hemost. 2018, 24, 780-789. [CrossRef] [PubMed]

4. Cahill, T.J.; Kharbanda, R.K. Heart failure after myocardial infarction in the era of primary percutaneous coronary intervention: Mechanism, incidence and identification of patients at risk. World J. Cardiol. 2017, 9, 407-415. [CrossRef]

5. Aimo, A.; Castiglione, V.; Borrelli, C.; Saccaro, L.F.; Franzini, M.; Masi, S.; Emdin, M.; Giannoni, A. Oxidative stress and inflammation in the evolution of heart failure: From pathophysiology to therapeutic strategies. Eur. J. Prev. Cardiol. 2020, 27, 494-510. [CrossRef]

6. Heusch, G.; Gersh, B.J. The pathophysiology of acute myocardial infarction and strategies of protection beyond reperfusion: A continual challenge. Eur. Heart J. 2017, 38, 774-784. [CrossRef] [PubMed]

7. Menasché, P.; Vanneaux, V. Stem cells for the treatment of heart failure. Curr. Res. Transl. Med. 2016, 64, 97-106. [CrossRef] [PubMed]

8. Kim, G.H.; Uriel, N.; Burkhoff, D. Reverse remodelling and myocardial recovery in heart failure. Nat. Rev. Cardiol. 2018, 15, 83-96. [CrossRef] [PubMed]

9. Yoshida, Y.; Yamanaka, S. Induced pluripotent stem cells 10 years later: For cardiac applications. Circ. Res. 2017, 120, 1958-1968. [CrossRef]

10. Goradel, N.H.; Hour, F.G.; Negahdari, B.; Malekshahi, Z.V.; Hashemzehi, M.; Masoudifar, A.; Mirzaei, H. Stem cell therapy: A new therapeutic option for cardiovascular diseases. J. Cell. Biochem. 2018, 119, 95-104. [CrossRef]

11. Morris, M.W., Jr.; Liechty, K.W. Cardiac progenitor cells in myocardial infarction wound healing: A critical review. Adv. Wound Care (New Rochelle) 2013, 2, 317-326. [CrossRef] [PubMed]

12. Oh, S.-W.; Lee, J.B.; Kim, B.; Jeon, S.; Kim, M.-K.; Nam, K.-H.; Ha, J.-R.; Bhatia, M.; Oh, G.T.; Kim, D.-Y. Peptidomimetic small-molecule compounds promoting cardiogenesis of stem cells. Arch. Pharmacal Res. 2012, 35, 1979-1988. [CrossRef] [PubMed]

13. Smart, N.; Bollini, S.; Dubé, K.N.; Vieira, J.M.; Zhou, B.; Davidson, S.; Yellon, D.; Riegler, J.; Price, A.N.; Lythgoe, M.F.; et al. De novo cardiomyocytes from within the activated adult heart after injury. Nature 2011, 474, 640-644. [CrossRef] [PubMed]

14. Takahashi, T.; Lord, B.; Schulze, P.C.; Fryer, R.M.; Sarang, S.S.; Gullans, S.R.; Lee, R.T. Ascorbic acid enhances differentiation of embryonic stem cells into cardiac myocytes. Circulation 2003, 107, 1912-1916. [CrossRef] [PubMed]

15. Willems, E.; Lanier, M.; Forte, E.; Lo, F.; Cashman, J.; Mercola, M. A chemical biology approach to myocardial regeneration. J. Cardiovasc. Transl. Res. 2011, 4, 340-350. [CrossRef] [PubMed]

16. Li, C.S.; Qu, Z.-Q.; Wang, S.-S.; Hao, X.-W.; Zhang, X.-Q.; Guan, J.; Han, F. Effects of Suxiao Jiuxin pill on oxidative stress and inflammatory response in rats with experimental atherosclerosis. J. Tradit. Chin. Med. 2011, 31, 107-111. [CrossRef]

17. Shang, Q.; Wang, H.; Li, S.; Xu, H. The effect of sodium tanshinone IIA sulfate and simvastatin on elevated serum levels of inflammatory markers in patients with coronary heart disease: A study protocol for a randomized controlled trial. Evid. Based. Complement. Alternat. Med. 2013, 2013, 756519. [CrossRef] [PubMed]

18. Wang, Y.; Wang, W.; Peng, W.; Liu, W.; Cai, W.; Xia, Z.; Zhang, H.; Xing, A.Z. Cardioprotective roles of the Chinese medicinal formula Bao-Xin-TANG on acute myocardial infarction in rats. Afr. J. Tradit. Complement. Altern. Med. 2017, 14, 65-74. [CrossRef] [PubMed]

19. Wang, Z.T.; Ng, T.B.; Yeung, H.W.; Xu, G.J. Immunomodulatory effect of a polysaccharide-enriched preparation of Codonopsis pilosula roots. Gen. Pharmacol. 1996, 27, 1347-1350. [CrossRef] 
20. Chang, K.-S.; Lee, N.-H.; Kuo, W.-W.; Hu, W.-S.; Chang, M.-H.; Tsai, F.-J.; Tsai, K.-H.; Yang, Y.-S.; Chen, T.-S.; Huang, C.-Y. Dung-Shen downregulates the synergistic apoptotic effects of angiotensin II plus Leu 27-IGF II on cardiomyoblasts. Acta Cardiol. Sin. 2014, 30, 56-66.

21. Luo, J.; Xu, H.; Chen, K. Systematic review of compound danshen dropping pill: A Chinese patent medicine for acute myocardial infarction. Evid. Based. Complement. Alternat. Med. 2013, 2013, 808076. [CrossRef] [PubMed]

22. Tsai, K.-H.; Lee, N.-H.; Chen, G.-Y.; Hu, W.-S.; Tsai, C.-Y.; Chang, M.-H.; Jong, G.-P.; Kuo, C.-H.; Tzang, B.-S.; Tsai, F.-J.; et al. Dung-shen (Codonopsis pilosula) attenuated the cardiac-impaired insulin-like growth factor II receptor pathway on myocardial cells. Food Chem. 2013, 138, 1856-1867. [CrossRef] [PubMed]

23. Gulick, J.; Subramaniam, A.; Neumann, J.; Robbins, J. Isolation and characterization of the mouse cardiac myosin heavy chain genes. J. Biol. Chem. 1991, 266, 9180-9185. [CrossRef]

24. Hescheler, J.; Fleischmann, B.; Lentini, S.; Maltsev, V.; Rohwedel, J.; Wobus, A.; Addicks, K. Embryonic stem cells: A model to study structural and functional properties in cardiomyogenesis. Cardiovasc. Res. 1997, 36, 149-162. [CrossRef]

25. Pucéat, M. Protocols for cardiac differentiation of embryonic stem cells. Methods 2008, 45, 168-171. [CrossRef] [PubMed]

26. Kan, C.D.; Lee, H.L.; Yang, Y.J. Cell transplantation for myocardial injury: A preliminary comparative study. Cytotherapy 2010, 12, 692-700. [CrossRef]

27. Morkin, E. Control of cardiac myosin heavy chain gene expression. Microsc. Res. Tech. 2000, 50, 522-531. [CrossRef]

28. Katano, W.; Moriyama, Y.; Takeuchi, J.K.; Koshiba-Takeuchi, K. Cardiac septation in heart development and evolution. Dev. Growth Differ. 2019, 61, 114-123. [CrossRef]

29. Sylva, M.; van den Hoff, M.J.; Moorman, A.F. Development of the human heart. Am. J. Med. Genet. A 2014, 164A, 1347-1371. [CrossRef] [PubMed]

30. Kawai, T.; Takahashi, T.; Esaki, M.; Ushikoshi, H.; Nagano, S.; Fujiwara, H.; Kosai, K.-I. Efficient cardiomyogenic differentiation of embryonic stem cell by fibroblast growth factor 2 and bone morphogenetic protein 2. Circ. J. 2004, 68, 691-702. [CrossRef]

31. Schneider, M.D.; Gaussin, V.; Lyons, K.M. Tempting fate: BMP signals for cardiac morphogenesis. Cytokine Growth Factor Rev. 2003, 14, 1-4. [CrossRef]

32. Armiñán, A.; Gandía, C.; Bartual, M.; García-Verdugo, J.M.; Lledó, E.; Mirabet, V.; Llop, M.; Barea, J.; Montero, J.A.; Sepúlveda, P. Cardiac differentiation is driven by NKX2.5 and GATA4 nuclear translocation in tissue-specific mesenchymal stem cells. Stem Cells Dev. 2009, 18, 907-918. [CrossRef] [PubMed]

33. Desjardins, C.A.; Naya, F.J. The function of the MEF2 family of transcription factors in cardiac development, cardiogenomics, and direct reprogramming. J. Cardiovasc. Dev. Dis. 2016, 3, 26. [CrossRef] [PubMed]

34. Hidaka, K.; Lee, J.; Kim, H.S.; Ihm, C.H.; Iio, A.; Ogawa, M.; Nishikawa, S.; Kodama, I.; Morisaki, T. Chamber-specific differentiation of Nkx2.5-positive cardiac precursor cells from murine embryonic stem cells. FASEB J. 2003, 17, 740-742. [CrossRef] [PubMed]

35. Koyanagi, M.; Haendeler, J.; Badorff, C.; Brandes, R.P.; Hoffmann, J.; Pandur, P.; Zeiher, A.M.; Kühl, M.; Dimmeler, S. Noncanonical Wnt signaling enhances differentiation of human circulating progenitor cells to cardiomyogenic cells. J. Biol. Chem. 2005, 280, 16838-16842. [CrossRef]

36. Pandur, P.; Läsche, M.; Eisenberg, L.M.; Kühl, M. Wnt-11 activation of a non-canonical Wnt signalling pathway is required for cardiogenesis. Nature 2002, 418, 636-641. [CrossRef]

37. Shaito, A.; Thuan, D.T.B.; Phu, H.T.; Nguyen, T.H.D.; Hasan, H.; Halabi, S.; Abdelhady, S.; Nasrallah, G.K.; Eid, A.H.; Pintus, G. Herbal medicine for cardiovascular diseases: Efficacy, mechanism, and safety. Front. Pharmacol. 2020, 11, 422. [CrossRef]

38. Kin, J.H. Cardiovascular diseases and panax ginseng: A review on molecular mechanisms and medical applications. J. Ginseng Res. 2012, 35, 16-26. [CrossRef]

39. Zhou, R.; Zhang, L. Search for a pharmacological role of Dangshen in alimentary system. J. Beijing Tradit. Chin. Med. 1991, 14, 47-48.

40. Tingyu, F.; Bo, J.; Qing, W. New Chinese-English Dictionary of Traditional Chinese Medicine, 2nd ed.; Chinese Medical Science and Technology Press: Beijing, China, 2013; ISBN 978-7506760553.

41. Liu, J.H.; Bao, Y.M.; Song, J.J.; An, L.J. Codonopsis pilosula (Franch) Nannf total alkaloids potentiate neurite outgrowth induced by nerve growth factor in PC12 cells. Acta. Pharmacol. Sin. 2003, 24, 913-917. 